graphic types with insufficiency or hyperfunction of certain of these glands. The thyroid is the gland of 'emotion' and ' rapidity' (writing very unequal, rapid, sometimes jerky, pointed and sharp) and the suprarenal is the gland of 'intensity' and of 'sustained courage' (writing firm, maintained, rather angular). With suprarenal insufficiency handwriting becomes light, soft, frequently small, sinuous, and sometimes tending to incline downwards. Hypothyroidals have slow, hesitating and round writing. Certain graphic signs are common to the functional disorders of different glands ; thus, writing which shows irregular formation of the strokes in such letters as $b, 1$, or $t$, has been observed in the ovarian insufficiency of puberty and the menopause and sometimes in parathyroid hypofunction.

It goes without saying that we must interpret such results with very considerable caution.

C. S. R.

\title{
Depchopatbology. PSYCHOLOGY.
}

[151] The effect of attitude upon feeling.-E. Francis Wells. Amer. Jour. Psychol., 1930, xlii, 571.

WHEN studying affective experience under a general instruction to describe the total, the observer's initial attitude tends to vary widely and unpredictably. Three types are described; the critical affective, the critical perceptive, and the common-sense attitude. The critical affective attitude is to react affectively to the stimulus and to observe critically the total experience. The critical perceptive attitude is to observe the stimulus-experience critically without reacting affectively to it. The common-sense attitude is that with which we take experience in everyday life as opposed to the critical laboratory set.

Experimental results show that the attitude with which one approaches an affective situation plays an important part in determining one's reaction to it. The nature of the affective reaction varies widely under different attitudes.

C. S. R.

\section{NEUROSES AND PSYCHONEUROSES.}

[152] Movement and types in children.-C. L. C. Burns. Psyche, 1930, xi, 63.

AN attempt has been made to observe a certain number of nervous children, part of whose treatment consists in attending a class in Margaret Morris movement. The following main differentiation has been made :

1. In certain children all the elements of movement are disintegrated -jerky, excessive, or inco-ordinated.

2. In another type, time seems to be affected more than balance, and there is poor, jerky rhythm. 